Colloque C2, suppl. au Journal de Physique II, Vol. 1, septembre 1991

\title{
THE APPLICATION OF A SUPERSONIC MOLECULAR BEAM SCATTERING SYSTEM TO UNDERSTANDING CVD PROCESSES
}

\author{
W. AHMED, J.S. FOORD (1), N.K. SINGH(1) and R.D. PILKINGTON \\ Department of Electronic and Electrical Engineering, \\ University of Salford, GB-Salford M5 4WT, Great-Britain
}

\begin{abstract}
A detailed description of a supersonic molecular beam scattering system for investigating surface processes involved in chemical vapour deposition (CVD) is given. The system can be used for both the carrying out of depositions and the simultaneous study of reaction mechanisms using modulated molecular beam relaxation spectroscopy and other surface science techniques. The advantages of this system for real time in-situ analysis of reaction mechanisms compared to previous approaches are discussed. The application of this system to the deposition and study of GaAs is discussed. With the increasing demand for improved layer quality, it is becoming more important to develop, control and understand the new generation of CVD processes.
\end{abstract}

\section{INTRODUCTION}

The deposition of thin films using chemical vapour deposition (CVD) is now a key technology which plays a critical role in several different industries including microelectronics, optical coating and surface finishing [1,2]. The most recent research and development has been driven by the demands of the microelectronics industry where a diverse range of materials including silicon, silicon nitride, gallium arsenide and indium phosphide are being used.

CVD is a versatile technique for the growth of thin films in which gaseous compounds are decomposed within a reactor leading to the formation of thin films and volatile decomposition products. There are several types of CVD technologies, which include atmospheric pressure, low pressure, plasma and photon enhanced, and metallorganic CVD. In the past, fortunately for most industrial processes, it was not essential to have a complete understanding of the deposition kinetics and thermodynamics considerations to produce good quality thin films for device manufacture. Most CVD processes in the microelectronics industry were optimised empirically by measuring the effects of reactor parameters on film characteristics and then making the necessary adjustments to produce the desired film properties. However, as device dimensions are rapidly shrinking, a detailed understanding of the reaction mechanisms and the kinetics associated with the CVD deposition process is becoming increasingly important.

All CVD processes involve a number of sequential steps, including mass transport, diffusion, adsorption, decomposition, and desorption. These can be grouped into two major stages, the mass transport stage and the kinetic (surface) stage. Important thin film growth requirements are film uniformity and growth rates. These can be controlled by suitable reactor design, by the inclusion of specially designed inlet injector systems (to ensure that the precursor molecules are delivered to the substrate surface at a rate far

(1) Research carried out at : Department of Inorganic

Chemistry, University of Oxford, South Parks Road,

GB-Oxford OXI 3QR, Great-Britain 
exceeding the rate of decomposition), but more commonly by adjustments to the process parameters, such as temperature, pressure, precursor gas flow rates etc [3-6].

The characterization of thin films grown under various process conditions yields a great deal of useful information regarding the overall CVD growth process, but the acquisition of information regarding the individual process stages is difficult. One approach to the separation of the mass transport and kinetic mechanisms is to theoretically model the mass transport by use of complex numerical modelling using modern computer methods [7] and then use this information in conjunction with the experimental data to obtain kinetic results. A second approach is to analyse the kinetic steps experimentally, but due to the nature of the CVD process i.e. the experimental conditions, it is not normally possible to separate the rate of mass transport and the kinetic process.

In this paper we will report a new approach to the experimental evaluation of the kinetic processes occurring during growth using the technique of modulated supersonic molecular beam relaxation spectroscopy and other surface analysis techniques. This is achieved under ultra high vacuum (UHV) conditions. The major advantages of working at UHV conditions is that mass transport is greatly enhanced and that all reactions are limited to the surface kinetic phase. The chemical information obtained should enable rapid development of CVD processes, give a more precise surface information and add greater meaning to the modelling currently being done.

\section{EXPERIMENTAL}

The technique used for probing the reactions involved is modulated molecular beam relaxation spectroscopy (MBRS), described previously for the investigation of MBE growth processes [8]. The experiment in its simplest form involves the generation of a molecular beam and the synchronous detection of products of surface scattering by mass spectrometry. Analysis of the scattered waveforms can then be carried out to determine reaction probabilities, reaction products, orders of reaction, and surface lifetimes of impinging species. As we will show later, this information can be used to determine the reaction pathways involved in the growth processes in CVD.

An earlier experimental approach, used successfully for MBE [8], was to employ a typical MBE set up, in which K-cells were used to generate the molecular beams within the UHV growth chamber, and modulated signal detection, achieved by the use of a chopping disc between either the K-cells and the sample or between the sample and the quadrupole mass spectrometer. This set up has certain limitations as it provides very little control over the quantum states of the incident beam which play an important role in determining the outcome of surface scattering events. It also relies heavily on the cryo-pumping efficiency of the MBE reactants and products of reaction to avoid spurious signals arising from stray molecules entering the quadrupole mass spectrometer.

Another approach involves the introduction of a small quantity of precursor into a UHV system containing a secondary ion mass spectrometry (SIMS), auger electron spectroscopy (AES) and mass spectrometry to carry out thermal desorption studies (TDS) [9]. This approach has several disadvantages including the lack of control over the precursor/sample interaction, background signals, and real time monitoring.

In order to avoid these problems we have adopted a new approach which utilises a highly collimated thermal or supersonic modulated molecular beam source, illustrated in figure 1. The MB660 molecular beam source consists of two separate vacuum stages each pumped by a diffusion rotary combination, gas admission system, rotating beam chopper, nozzle, skimmer, and aperture for beam collimation. The nozzle can be heated to $1000 \mathrm{~K}$ and cooled to $200 \mathrm{~K}$ and is designed to produce uniform heating. Its aperture (50 


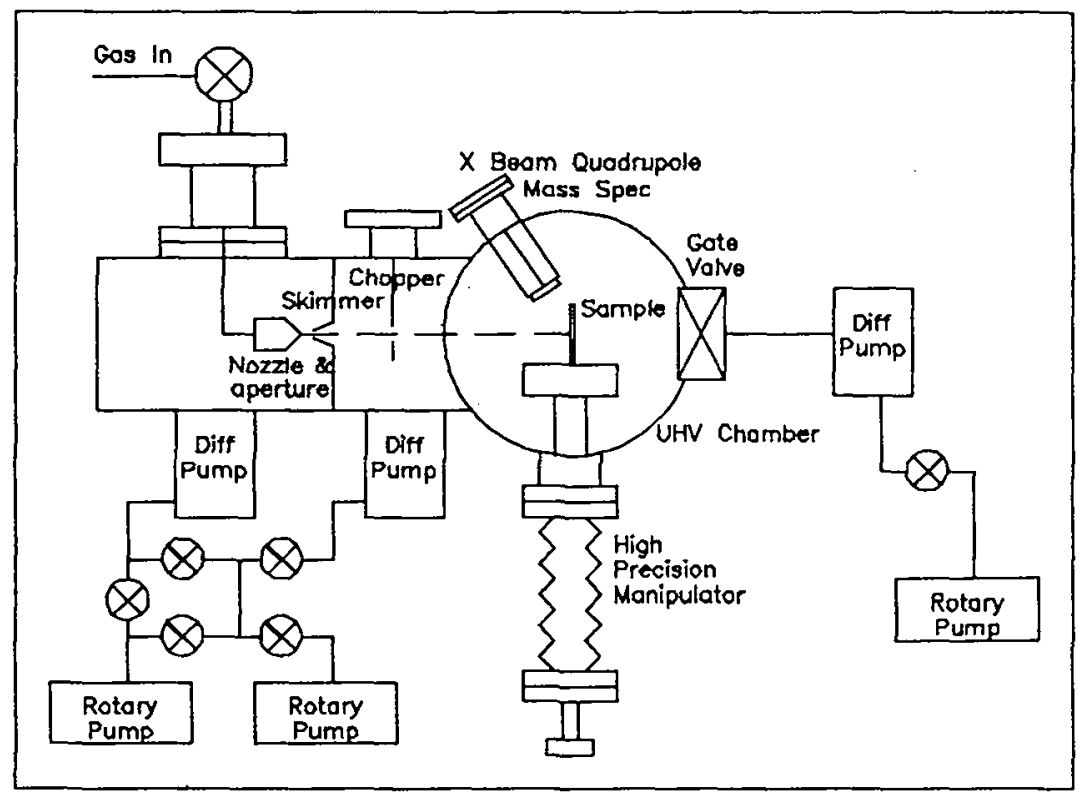

Figure 1 Schematic of the molecular beam scattering system.

micron) is laser drilled, made from platinum, and the complete assembly is mounted on a $\mathrm{XYZ}$ manipulator for ease of alignment. This generates a supersonic molecular beam by the expansion of the desired precursor from a stagnant region, behind it, through the aperture into the first vacuum stage. The cone shaped skimmer has an orifice of $0.5 \mathrm{~mm}$ diameter and this helps to shape the molecular beam and prevent the formation of shock waves which can destroy the beam. The skimmer is mounted on an XY stage with a $5 \mathrm{~mm}$ movement in both directions. The rotating chopping disc, located in the second vacuum stage, consists of an aluminium disc and a 3 phase synchronous motor capable of rotating at speeds up to $400 \mathrm{~Hz}$.

The supersonic beam source is found to generate fluxes of $5 \times 10^{18}$ atoms/steradian/second with helium and a translational energy spread of $<1 \%$ when operated with a stagnant He pressure of 1-10bar. Typical operating pressures are $2 \times 10^{-3} \mathrm{mbar}, 5 \times 10^{-5} \mathrm{mbar}$ and $2 \times 10^{-10} \mathrm{mbar}$ in the first, second and growth stages respectively. The beam is approximately $4 \mathrm{~mm}$ in diameter at the sample.

The growth chamber has a separate diffusion/rotary pump combination and is fitted with a liquid nitrogen cooled inner shroud for cryo-pumping. Mass spectral analysis is carried out using a 400amu quadrupole mass spectrometer fitted with a cross beam ion source and associated electronics for pulse counting. A multi channel analyser is used to accumulate counts in sequential time channels for time of flight studies and is referenced to the slots in the beam modulator discs. The mass spectrometer in its standard configuration is mounted on a linear motion drive, which enables the monitoring of both the incident and the reflected beam; it has its own liquid nitrogen shroud which provides additional differential pumping. Alternately the mass spectrometer can be mounted in the vertical plane on a different flange with active differential pumping and beam chopper assembly for modulation of the detected products of deposition.

The growth chamber is fitted with three other DC gas sources, one of which incorporates a tantalum cracker for the decomposition of group $\mathrm{V}$ hydrides. It is thus possible, for example, to carry out 
experiments where a range of DC molecular beams are impinging simultaneously onto the sample and to examine the signal response arising from the modulation of one of the chemicals involved. The sample is mounted on a high precision linear translator which provides $360 \mathrm{deg}$ sample rotation and sample heating up to $1200 \mathrm{~K}$ This manipulator also enables the sample to be translated via an isolation valve into a separate surface analysis chamber equipped with RFA for LEED and Auger studies and $\mathrm{Ar}^{+}$ion etching facilities. Other techniques such as SIMS, XPS, HREEL etc. can be readily incorporated.

This configuration provides a flexible approach to research into the development of CVD and other thin film growth processes. The reduced gas load (of the precursor) in the main chamber permits effective surface pressures of $<10^{-6}$ mbar whilst background pressures remain $<5 \times 10^{-10}$ mbar. The modulated beam can be generated at thermal translational energies using the effusive source or an unseeded supersonic expansion. Alternately the energy can be varied by heating and cooling the nozzle or by the use of beam seeding techniques [10]. The use of a modulated beam permits the investigating of chemical kinetics, the DC beams permit conventional growth runs to be carried out with translation into the surface analysis chamber permitting the immediate analysis of the films grown.

\section{SYSTEM PERFORMANCE AND APPLICATION TO THE CVD OF GaAs}

The chopping disc in the second stage defines the nature of the beam modulation. Two types of modulation are achieved by the translation of the disc in the direction at right angles to the molecular beam. The disc has cut-outs which provides an approximate delta modulation function or a rectangular function with equal on and off periods.

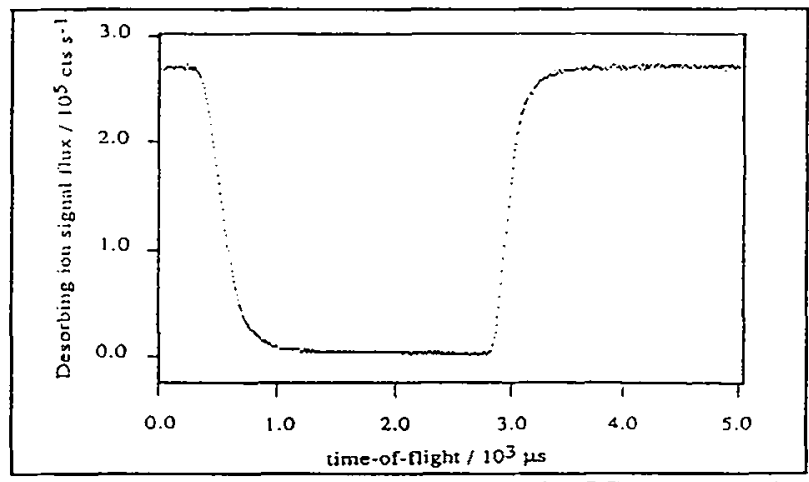

Figure 2 Straight through spectrum of TEG - rectangular modulation

The spectrum obtained using a supersonic expansion of triethylgallium (TEG) monitoring a straightthrough beam is plotted in figure 2 . Features to be noted particularly are the well behaved waveform obtained and the rapid rise and decay times. The former arises directly from the excellent spatial and time resolution which eliminates unwanted background signals. The latter is consistent with a velocity spread of $<1 \%$ arising from supersonic expansion. Figure 3 shows the scattered TEG from GaAs substrates using an effusive beam. The spread in the time of flight spectrum is due to the finite residence time involved in the scattering process. The use of a delta modulation function with an effusive source is shown in figure 4. Once again excellent waveforms are obtained with the absence of unwanted background. 


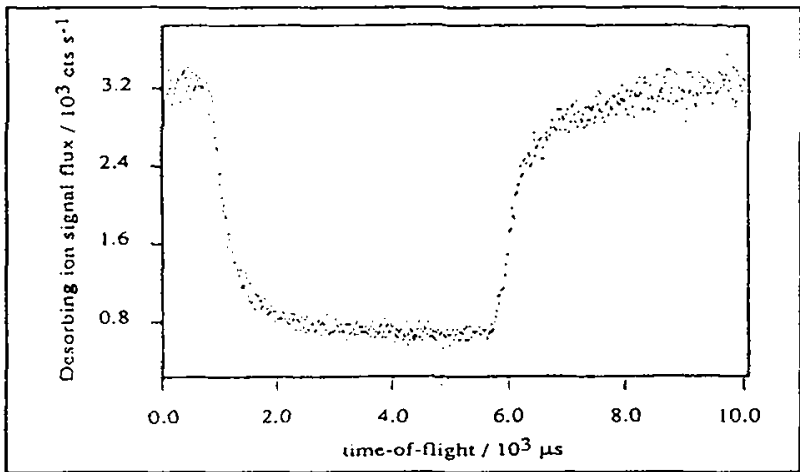

Figure 3 Spectrum of TEG reflected from GaAs - delta modulated

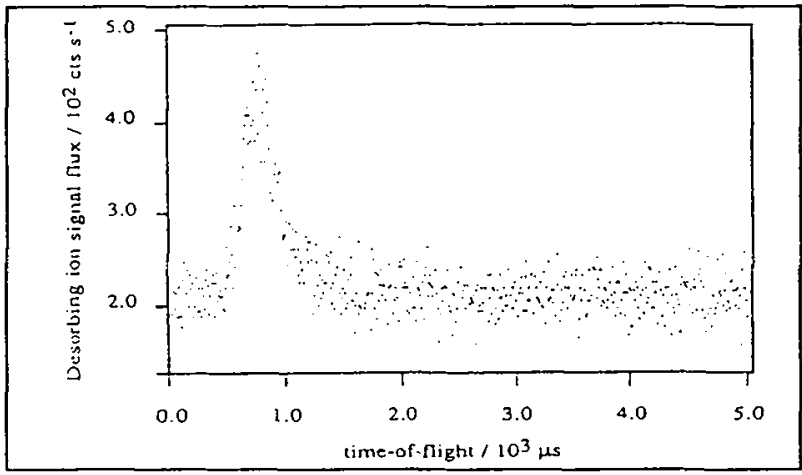

Figure 4 Spectra for TEG reflected from $\mathrm{GaAs}$ using an effusive beam

The most direct information to emerge from modulated molecular beam studies is the identification of the products of surface scattering.

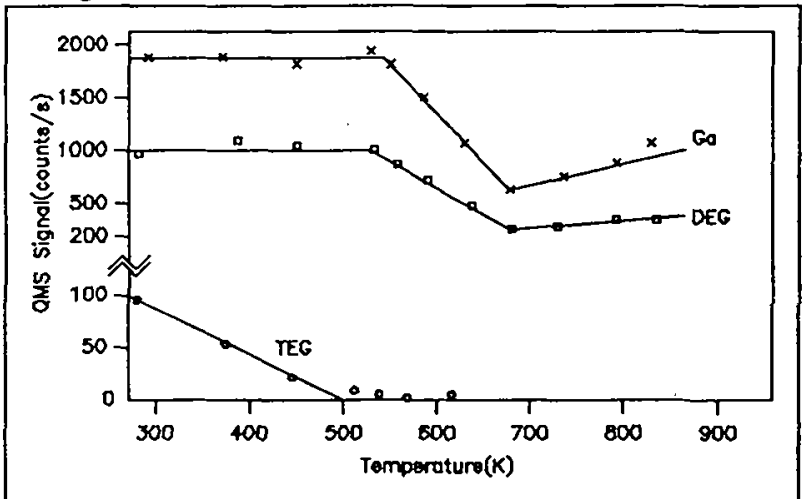

Figure 5 Thermal desorption spectra of reaction products resulting from a TEG beam impinging on GaAs 


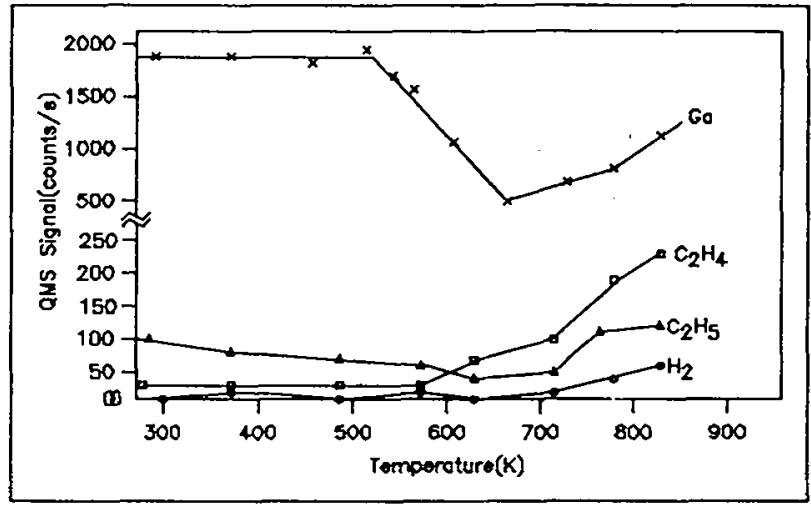

Figure 6 Thermal desorption spectra of reaction products resulting from a TEG beam impinging on GaAs

In figures 5 and 6 we present results of the first modulated studies which have been carried out on the growth of GaAs using $\mathrm{As}_{2}$ and TEG. At low temperatures the incident TEG flux reflects from the surface as mainly diethylgallium (DEG) with some TEG also being detected. As the temperature is raised above $600 \mathrm{~K}$ the reflected DEG flux suddenly drops before increasing slowly at even higher temperatures. Monitoring of the reaction products from TEG cracking shows that a mixture of ethene, hydrogen and ethane are present. This data can be understood in the light of our previous work [11].

At low temperatures the GaAs substrate is covered in a strongly bound layer of adsorbed species which inhibits film growth. However the surface is still reactive in that significant TEG is converted to DEG via the following reaction:

$$
\left(\mathrm{C}_{2} \mathrm{H}_{5}\right)_{3} \mathrm{Ga}(\mathrm{g}) \longrightarrow\left(\mathrm{C}_{2} \mathrm{H}_{5}\right)_{2} \mathrm{Ga}(\mathrm{g})+\mathrm{C}_{2} \mathrm{H}_{5}(\mathrm{~g})
$$

As the sample temperature is raised above $550 \mathrm{~K}$ the strongly bound species start to desorb exposing active surface sites on the GaAs substrate. These sites drive the following reactions:

$$
\begin{aligned}
\left(\mathrm{C}_{2} \mathrm{H}_{5}\right)_{3} \mathrm{Ga}(\mathrm{g}) & \longrightarrow\left(\mathrm{C}_{2} \mathrm{H}_{5}\right)_{3} \mathrm{Ga}(\mathrm{a}) \\
\left(\mathrm{C}_{2} \mathrm{H}_{5}\right)_{3} \mathrm{Ga}(\mathrm{a}) & \longrightarrow\left(\mathrm{C}_{2} \mathrm{H}_{5}\right)_{2} \mathrm{Ga}(\mathrm{a})+\mathrm{C}_{2} \mathrm{H}_{5}(\mathrm{a}) \\
\left(\mathrm{C}_{2} \mathrm{H}_{5}\right)_{2} \mathrm{Ga}(\mathrm{a}) & \longrightarrow \mathrm{Ga}(\mathrm{a})+2\left(\mathrm{C}_{2} \mathrm{H}_{5}\right)(\mathrm{a}) \\
\mathrm{C}_{2} \mathrm{H}_{5}(\mathrm{a}) & \longrightarrow \mathrm{C}_{2} \mathrm{H}_{5}(\mathrm{~g}) \\
\mathrm{C}_{2} \mathrm{H}_{5}(\mathrm{a}) & \longrightarrow \mathrm{C}_{2} \mathrm{H}_{4}(\mathrm{~g})+\mathrm{H}_{2}(\mathrm{~g}) \\
\left(\mathrm{C}_{2} \mathrm{H}_{5}\right)_{2} \mathrm{Ga}(\mathrm{a}) & \longrightarrow\left(\mathrm{C}_{2} \mathrm{H}_{5}\right)_{2} \mathrm{Ga}(\mathrm{g})
\end{aligned}
$$

Reaction (1) results in the formation of DEG (even at low temperatures) when no growth takes place. Reactions (2)-(6) results in the net deposition of $\mathrm{Ga}$ on the surface which subsequently reacts with arsenic species resulting in the growth of GaAs. These reactions manifest themselves in the decay of the reflected $\mathrm{Ga}$ alkyl signal intensities in the $500-700 \mathrm{~K}$ range. Finally reaction (7) represents the high temperature 
desorption of the DEG intermediate in a process which competes with the overall cracking.

This work provides an illustration of the way in which modulated molecular beam reactive scattering may be used to obtain an insight into the CVD of GaAs. It also shows the molecular origins of the problems encountered in CVD production processes. The results obtained may be fed into future modelling efforts to obtain a better understanding of the overall CVD mechanisms.

\section{REFERENCES}

1. Kennedy L W, Smith D E and Mccaughan D V, GEC J. Sci. and Techn. 48(2) (1982) 90

2. Rosler R S, Solid State Techn. 20(4) (1977) 63

3. Ahmed W and Meakin D B, J. Crystal Growth, 79 (1986) 394

4. Jensen K F, Hitchman M L and Ahmed W, Proc 5th Int. conf on CVD, Upsala, Sweden, (1984) 144

5. Meyerson B S and Olbricht W, J. Electrochem. Soc. 131 (1984) 2365

6. Learn A J, Proc. Conf. on Semiconductor and Integrated Techn., University of California, Berkeley, USA, (1986) 709

7. Jensen K F and Graves D B, J. Electrochem Soc. 131 (1983) 1950

8. . Foxon C T, Boundry M R and Joyce B A, Surface Science, 44 (1974) 69

9. Meyerson B S and Yu M L, J. Electrochem. Soc. 131 (1984) 2369

10. Whitehead C, European Spectroscopy News, 57 (1984) 10

11. Wee A T, Singh N K, Murrell A J, Foord J S, Andrews D A and Davies G J, Submitted to J. Applied Physics. 